\section{January 2000}

Dental Expo 2000 and 3ri Triennial Commonwealt] Dental Association \& 54th Indian Dental Association Congress \& Exhibition

Date: $28.01 .00-01.02 .00$

Venue: New Delhi, India

Contact: Congress Secretariat

CDA-IDA Congress 2000,

C-56, South Extension Part-II, New Delhi 110 049,

India

Tel: +91 11625 1823/0169/2398/5918

Fax: +91116256688

E-mail: cda.ida2000@vsnl.com

\section{February 2000}

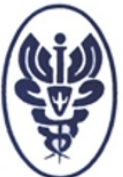
he Barbados Dental Association 12th Annual Mid-Winter Convention Date: $17.02 .00-20.02 .00$ enue: Christ Church Contact: Mrs Fay Mack,

17 Pine Road, Belleville,

St Michael, Barbados,

West Indies.

Tel: +2462286488

Fax: +2462286488

\section{March 2000}

Academy of Laser Dentistry's Seventh Annual Conference and Exhibition

Date: $01.03 .00-04.03 .00$

Venue: Panama City Beach, Florida, USA

Contact: Gail S Siminovsky

Executive Director,

Academy Of Laser Dentistry, 9629 Westview Drive

Coral Springs, Hl 33076, USA

Tel: +1 9543463776

E-mail: laserexec@laserdentistry.org

GKT Dental Institute: Clinical Day At Guy's Dental Hospital

Date: 04.03 .00

Venue: London

Contact: Mrs S Biden

Tel: +44 (0)20 79554587

HC2000: Healthcare Computing

Conference \& Exhibition

Date: 20.03 .00 - 22.03.00

Venue: Harrogate

Contact: Fiona Campbell, BJHC,

45 Woodland Grove,

Weybridge,

Surrey KT13 9EQ

Tel: $+44(0) 1932821723$

Fax:+44 (0) 1932820305

Website: www.healthcare-computing.co.uk

The 2000 Meeting Of The Craniofacial

Society Of Great Britain

Date: 22.03 .00 - 24.03.00
Venue: Reading

Contact: The Courses and Conference

Office, Institute Of Child Health,

30 Guilford Street,

London WC1N $1 \mathrm{EH}$

Tel: +44 (0)20 7829 8692/020 78138394

Fax: +44 (0)207831 6902

E-mail: Courses@ich.ucl.ac.uk

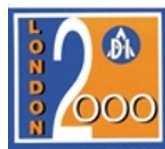

London 2000: International Congress on Implant Dentistry

Date: 31.03 .00 - 01.04.00

Venue: London Heathrow Marriott Hotel

Contact: Cherry Wilson,

Association of Dental Implantology UK

37 Halford Road, Richmond,

Surrey TW 106 AW

Tel: +44 (0)20 83320321

Fax: +44 (0)20 89400337

E-mail: congress@fdi.org.uk

Website: www.fdi.org.uk/worldenta

\section{April 2000}

BDA Conference: Dentistr 2000 - The Millennium

celebration for oral care

Date: $06.04 .00-08.04 .00$

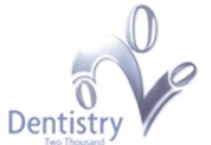

Venue: Birmingham

Contact: Charlotte Long,

BDA Conference Office,

64 Wimpole Street, London W1M 8AL

Tel: +44 (0)20 79350875 ext 286

Fax: +44 (0)2074860855

DPB Dental Conference and Open

Meeting

Date: 17.4 .00 - 18.4 .00

Venue: Eastbourne

Contact: Heather Deacon

Tel: $+44(0) 1323433307$

Fax:+44 (0) 1323433432

E-mail: pr@dentanet.org.uk

\section{May 2000}

9th International Dental Congress On Modern Pain Control: A new millennium in analgesia, sedation and anaesthesia Date: 02.05 .00 - 05.05.00

Venue: Jersualem, Israel

Contact: 9th IFDAS Secretariat,

International Travel and Congresses Ltd,

P.O. Box 29313 ,

20 Rothschild Boulevard,

Tel Aviv 61292, Israel

Tel: +972 37951444

Fax: +97235107716

E-mail:congs@internationaltc.co.il

Website: www6.huji.ac.il/ dental/news.htm

\section{CONSEURO 2000}

Date: 11.05 .00 - 13.05.00

Venue: Palazzo della Cultura e dei

Congressi, Bologna, Italy

Contact: Dr Margaret Wilson,

Restorative Dentistry,
University Dental Hospital of Manchester, Higher Cambridge Street,

Manchester M15 6FH.

Tel: +44 (0)1612756619

Fax: +44 (0)161 2756710

E-mail: wilson@fs1.den.man.ac.uk

\section{October 2000}

New Zealand Dental Association Biennial Conference

Date: 04.10 .00 - 08.10 .00

Venue: Dunedin, New Zealand

Contact: NZDA Conference,

PO Box 1029, Dunedin,

New Zealand

Tel: +643477 1377

Fax: +6434772720

E-mail: dcms@dcms.co.nz

\section{November 2000}

FDI World Dental Congress 2000

Date: $29.11 .00-02.12 .00$

Venue: Paris

Contact: FDI, 7 Carlisle Street,

London W1V 5RG

Tel: +44 (0)2079357852

Fax: +44 (0)20 74860183

Website: www.fdi.org.uk

International Dental

Film Festival

Date: 29.11 .00 - 02.12 .00

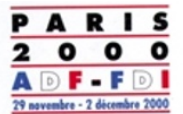

Venue: Paris

Contact: Association Dentaire Francaise, 6 Rue Guillaume Tell 75017, Paris.

Tel: +33144010270

Fax: +331 47639028

E-mail:adf@adf.asso.fr

Website: www.adf.asso.fr

\section{1}

\section{May 2001}

30th Australian Dental Congress

Date: 04.05.01-08.05.01

Venue: Brisbane Convention \&

Exhibition Centre,

Brisbane, Australia

Contact: Secretariat,

30th Australian Dental Congress,

P.O. Box 1280 Milton,

Queensland 4064, Australia

Tel: +61733690477

Fax: +61 733691512

E-mail: ada2001@im.com.au
Please send details of forthcoming events to: Kate Craig,

British Dental Journal 64 Wimpole Street, London W1 M 8AL England 